\title{
Critical function of Bmx/Etk in ischemia- mediated arteriogenesis and angiogenesis
}

Yun He, ${ }^{1,2}$ Yan Luo, ${ }^{1,3}$ Shibo Tang, ${ }^{1,3}$ liro Rajantie, ${ }^{4}$ Petri Salven, ${ }^{4}$ Matthias Heil, ${ }^{5}$ Rong Zhang, ${ }^{1}$ Dianhong Luo, ${ }^{1}$ Xianghong Li, ${ }^{1}$ Hongbo Chi, ${ }^{1}$ Jun Yu, ${ }^{1}$ Peter Carmeliet, ${ }^{6}$ Wolfgang Schaper, ${ }^{5}$ Albert J. Sinusas, ${ }^{1}$ William C. Sessa, ${ }^{1}$ Kari Alitalo, ${ }^{4}$ and Wang Min ${ }^{1}$

${ }^{1}$ Interdepartmental Program in Vascular Biology and Transplantation, Yale University School of Medicine, New Haven, Connecticut, USA. ${ }^{2}$ School of Public Health, and ${ }^{3}$ Zhongshan Ophthalmic Center, Sun Yat-Sen University, Guangzhou, People's Republic of China. ${ }^{4}$ Molecular and Cancer Biology Program and Ludwig Institute for Cancer Research, Haartman Institute, Biomedicum Helsinki and Helsinki University Hospital, University of Helsinki, Helsinki, Finland. 5epartment of Experimental Cardiology, Max-Planck-Institute for Heart and Lung Research, Bad Nauheim, Germany. ${ }^{6}$ Center for Transgene Technology and Gene Therapy, Flanders Interuniversity Institute for Biotechnology (VIB), Leuven, Belgium.

\begin{abstract}
$\mathrm{Bmx} /$ Etk non-receptor tyrosine protein kinase has been implicated in endothelial cell migration and tube formation in vitro. However, the role of $\mathrm{Bmx}$ in vivo is not known. $\mathrm{Bmx}$ is highly induced in the vasculature of ischemic hind limbs. We used both mice with a genetic deletion of $\mathrm{Bmx}(\mathrm{Bmx}-\mathrm{KO}$ mice) and transgenic mice expressing a constitutively active form of $\mathrm{Bmx}$ under the endothelial Tie-2 enhancer/promoter (Bmx-SK-Tg mice) to study the role of $\mathrm{Bmx}$ in ischemia-mediated arteriogenesis/angiogenesis. In response to ischemia, Bmx-KO mice had markedly reduced, whereas Bmx-SK-Tg mice had enhanced, clinical recovery, limb perfusion, and ischemic reserve capacity when compared with nontransgenic control mice. The functional outcomes in these mice were correlated with ischemia-initiated arteriogenesis, capillary formation, and vessel maturation as well as Bmx-dependent expression/activation of TNF receptor 2 - and VEGFR2-mediated (TNFR2/VEGFR2-mediated) angiogenic signaling in both hind limb and bone marrow. More importantly, results of bone marrow transplantation studies showed that $\mathrm{Bmx}$ in bone marrow-derived cells plays a critical role in the early phase of ischemic tissue remodeling. Our study provides the first demonstration to our knowledge that $\mathrm{Bmx}$ in endothelium and bone marrow plays a critical role in arteriogenesis/angiogenesis in vivo and suggests that $\mathrm{Bmx}$ may be a novel target for the treatment of vascular diseases such as coronary artery disease and peripheral arterial disease.
\end{abstract}

\section{Introduction}

Peripheral arterial disease is a common disease found in $10 \%-25 \%$ of patients over the age of 55 years, and its occurrence and severity are strongly correlated with other cardiovascular risk factors that lead to coronary artery disease and stroke, such as hyperlipidemia, smoking, and endothelial dysfunction (1-3). An animal model has been created by ischemic injury of mouse hind limb, and studies using this mouse model reveal that a reduction in calf blood pressure ratios and blood flow correlate with a marked decrease in the number of capillaries surrounding each muscle fiber (capillary/fiber ratio), indicative of the inability to mount an adaptive angiogenic response $(4,5)$. Mechanistically, ischemiainduced vascular remodeling occurring in both human patients and animal models can be explained as follows: stenotic narrowing of the common ileac, femoral artery, or branch off the femoral artery leads to tissue ischemia, thereby creating regional hypoxia and mismatch of oxygen supply versus demand in skeletal myocytes. The reduction in tissue perfusion correlates with activation of vascular endothelial cells and the recruitment of inflammatory cells, which in turn secrete chemokines and cytokines that may participate in arterializing preformed collaterals (termed arteriogenesis) to provide a stable conduit for blood flow to the

Nonstandard abbreviations used: BMT, bone marrow transplantation; Bmx, bone marrow tyrosine kinase in chromosome X; EPC, endothelial progenitor cell; $\mathrm{PH}$, pleckstrin homology; PlGF, placental growth factor; qRT-PCR, real-time quantitative PCR; TNFR, TNF receptor.

Conflict of interest: The authors have declared that no conflict of interest exists. Citation for this article: J. Clin. Invest. 116:2344-2355 (2006). doi:10.1172/JCI28123. distal limb. Contemporaneously, redistribution of blood flow and the attendant changes in shear stress may collaborate with local cytokines to arterialize immature collaterals and perhaps induce angiogenesis. Once a stable collateral circulation has been established, the improvement in distal blood flow and shear stress triggers an increase in capillary angiogenesis, thus increasing capillary-to-fiber ratios and oxygen delivery to the dependent portions of the lower limb. Therefore, proportionally regulated arteriogenesis and angiogenesis are necessary to improve nutritive blood flow to tissue and promote functional limb salvage. The availability of $\mathrm{KO}$ or transgenic mice that are developmentally "normal" permits the testing of genes required for postnatal hind limb angiogenesis and remodeling. Thus, the ischemia hind limb model is a very useful tool for studying the function of genes critical for inflammatory arteriogenesis/angiogenesis $(4,5)$.

Bone marrow tyrosine kinase in chromosome $\mathrm{X}(\mathrm{Bmx}$; also called endothelial/epithelial tyrosine kinase [Etk]) is a member of the Tec family of non-receptor tyrosine kinases. Bmx and 3 other members of this family (Btk, Itk, and Tec) participate in signal transduction in response to growth factor receptors, cytokine receptors, $G$ protein-coupled receptors, antigen receptors, and integrins (6-13). They share a common domain structure including a pleckstrin homology $(\mathrm{PH})$ domain; a Tec homology $(\mathrm{TH})$ domain, which has a PXXP motif (with the exception of Bmx); an SH3 and an SH2 domain; and a kinase domain. Based on data from Bmx activation by focal adhesion kinase (FAK), it has been proposed that integrin-induced binding of Bmx to FAK leads to phosphorylation of Bmx at Y 40 to open up the "closed" conformation of the inactive $\mathrm{Bmx}$ and allow the kinase to be 
A

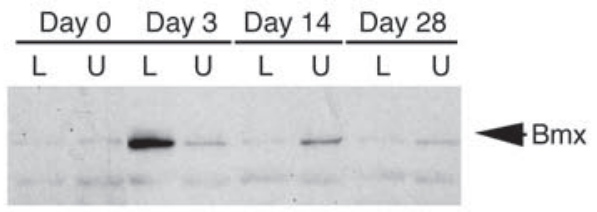

(IB: Bmx)

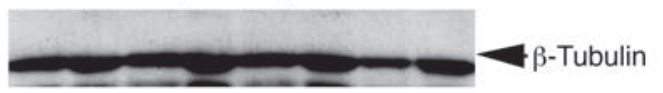

(IB: $\beta$-tubulin)
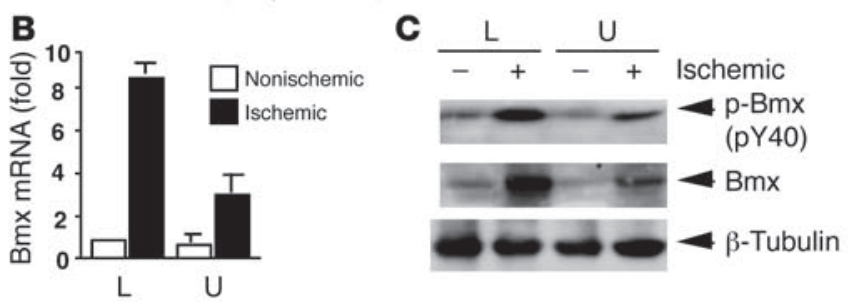

D
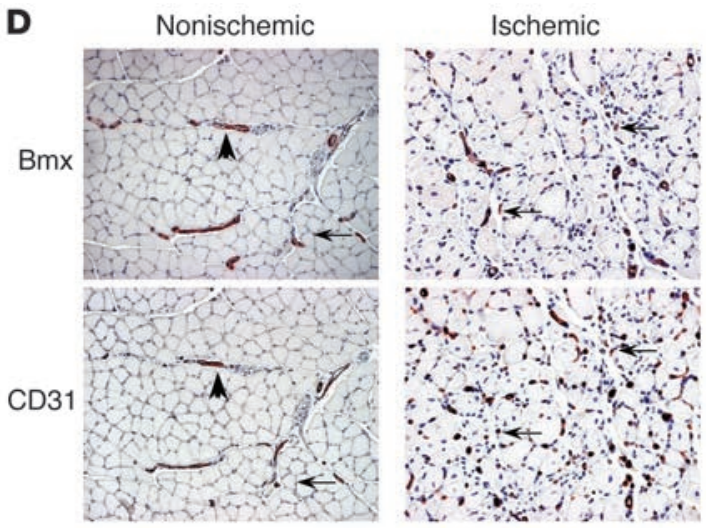

Figure 1

Ischemia induces upregulation of Bmx expression in vasculature and generation of EC-specific Bmx-Tg mice. (A) C57BL/6 ( $n=3$ ) mice were subjected to hind limb ischemia, and the upper $(U)$ and lower limbs $(L)$ were harvested on days $0,3,14$, and 28 after surgery, as indicated. Bmx protein expression was determined by Western blotting with anti-Bmx antibody. $\beta$-Tubulin protein was used for normalization. (B) Day 3 samples from $\mathbf{A}$ were used for Bmx mRNA expression as determined by qRT-PCR. Data represent the fold increases in Bmx mRNA with expression in nonischemic lower limb taken as 1.0. (C) Day 3 samples were used for Bmx phosphorylation as determined by Western blotting with anti-phospho-Bmx antibody (pY40). (D) Bmx is induced in vascular endothelium. Bmx and the EC marker CD31 in the frozen sections of nonischemic and ischemic (day 3) lower limb were detected by immunohistochemistry with anti-Bmx and anti-CD31 antibodies, respectively. Bmx expression in a large artery in the nonischemic section and capillaries in the ischemic tissue section are indicated by arrowheads and arrows, respectively.

phosphorylated by Src family kinases at the highly conserved tyrosine residue Y566 in the catalytic domain $(13,14)$. Several downstream effectors of Bmx involved in cell migration have been reported. Bmx through its $\mathrm{PH}$ domain directly binds to and activates RhoA (but not Rac1 and Cdc42) (15). Similarly, Bmx through its PH domain binds to and activates PAK1 (16), a $65-\mathrm{kDa}$ serine/threonine kinase implicated in integrin-induced EC migration and angiogenesis through modulation of EC contraction (17). We have shown that $\mathrm{Bmx}$ mediates induction by TNF of the PI3K/Akt angiogenic pathway (9), which has been well documented in growth factor-stimulated cell migration and angiogenesis (18-22). PI3K/Akt may induce angiogenesis by multiple downstream effectors including the Rho family of small GTPase, PAK1, and endothelial nitric oxide synthase $(18-21,23)$. However, the role of $\mathrm{Bmx}$ in vivo is not clear. We have previously shown that Bmx-deficient mice have normal embryonic vasculature (24). Furthermore, our recent data using mice in which the Bmx transgene is expressed specifically in epidermal keratinocytes suggest that Bmx may be involved in wound reepithelialization (25).

Although $\mathrm{Bmx}$ is primarily expressed in bone marrow and arterial endothelium in vivo, the roles of $\mathrm{Bmx}$ in arteriogenesis and angiogenesis in vivo are not known. In the present study, we used mice deficient of the $\mathrm{Bmx}$ gene $(\mathrm{Bmx}-\mathrm{KO})$ and mice expressing an endothelium-specific constitutively active form of $\mathrm{Bmx}$ (Bmx-SK-Tg) to define the role of Bmx in arteriogenesis and angiogenesis in the ischemia hind limb model. Our data suggest that Bmx plays a critical role in ischemia-induced arteriogenesis/angiogenesis by increasing collateral arterial growth, capillary formation, vessel maturation, and bone marrow-derived endothelial progenitor cell (EPC) mobilization, likely by activating TNF receptor 2/VEGFR2-dependent (TNFR2/VEGFR2dependent) angiogenic signaling pathways.

\section{Results}

Bmx is highly induced and activated in ischemic hind limbs. To determine the role of Bmx in inflammatory arteriogenesis/angiogenesis, we first examined Bmx expression and activation in response to ischemic injury in a mouse hind limb model induced by surgical arteriectomy of the left femoral artery. Upper and lower limbs of the ischemic (left) and nonischemic (right) legs were harvested on days 3,14 , and 28 after surgery, and the expression of Bmx protein was determined by Western blotting with anti-Bmx antibody. Bmx was highly induced (by 8-fold) in the ischemic lower hind limb, and the induction peaked at day 3 but declined to the basal level by day 14 (Figure 1A). Expression of Bmx in the ischemic upper hind limb was also weakly induced and persisted up to 2 weeks (Figure 1A). Day 3 samples were then used to determine Bmx mRNA induction by realtime quantitative PCR (qRT-PCR). Consistent with Bmx protein expression, Bmx mRNA was induced in both upper and lower parts of ischemic hind limb compared with the nonischemic hind limb (Figure 1B). To determine whether induced Bmx is active, Bmx activation was determined by Western blotting with phospho-specific antibody (anti-pY40). Results showed that Bmx phosphorylation was significantly upregulated in the ischemic hind limbs compared with the nonischemic tissues and Bmx induction and activation were greater in the lower limb (Figure 1C). To determine which cell type expressed Bmx in response to ischemia, Bmx expression was determined by immunohistochemistry with anti-Bmx antibody. Bmx was primarily induced in vascular endothelium including capillaries, which showed positive staining with anti-CD31 antibody by immunohistochemistry (Figure 1D, arrows). A control goat IgG did not show any staining (Supplemental Figure 1; supplemental material available online with this article; doi:10.1172/JCI28123DS1). In control experiments, Bmx expression was primarily detected in large arteries in nonischemic tissues (Figure 1D, arrowheads). 


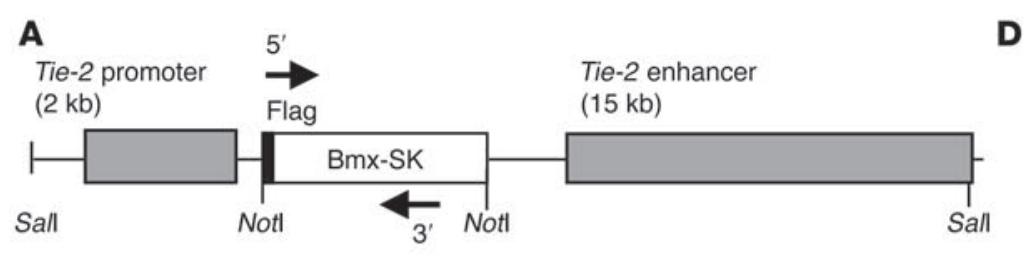

B
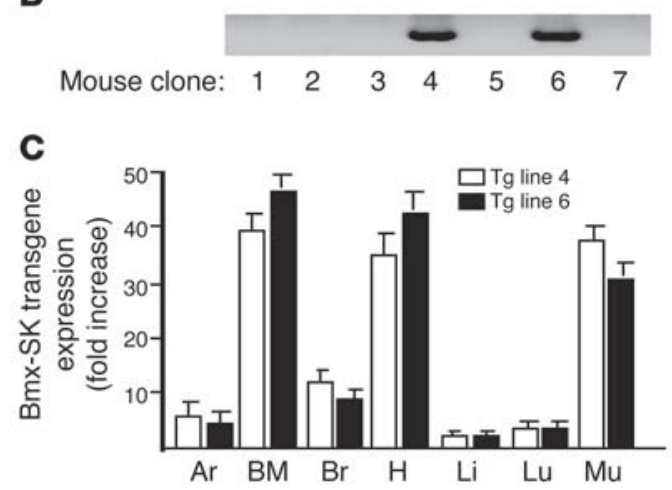

D

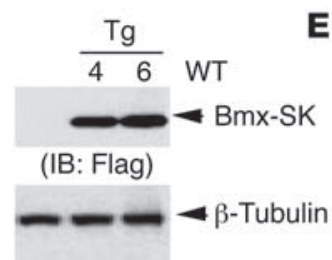

(IB: $\beta$-tubulin)

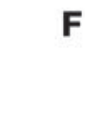

Bmx-SK

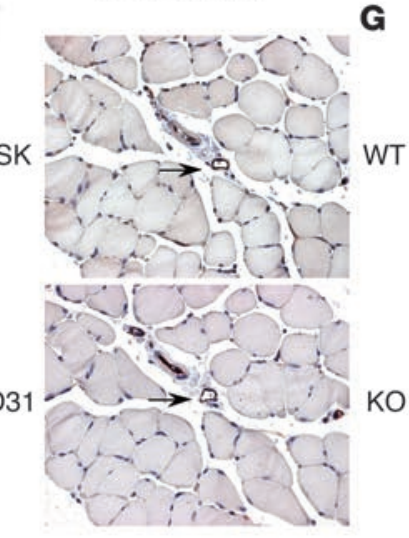

G
E WT Tg KO

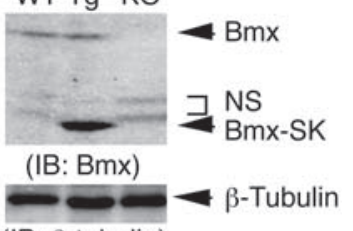

(IB: $\beta$-tubulin)

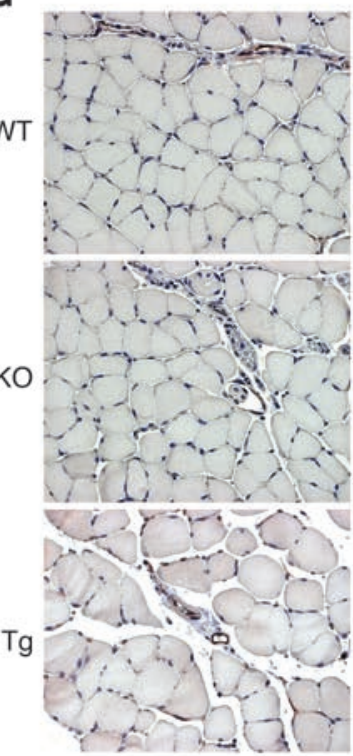

Figure 2

EC-specific transgenic mice: specific expression of Bmx-SK in ECs driven by Tie-2 promoter. (A) Schema of transgenic vector showing inserted Flag-tagged human Bmx-SK and location of primers used for genotyping by PCR. (B) Genotyping for Bmx-SK transgene. Tail genomic DNA was isolated, and genotyping was performed by PCR with specific primers as described in Methods. Lines 4 and line 6 were positive for Bmx-SK. (C) Tissue distribution of Bmx-SK expression. Bmx-SK mRNA in indicated tissues of Bmx-SK-Tg mice $(n=2$ for each line) was quantified by qRTPCR with 18S rRNA for normalization. Data are presented fold increase, with expression of Bmx-SK mRNA in liver taken as 1.0. Ar, aorta; Br, brain; H, heart; Li, liver; Lu, lung; Mu, muscle. (D) Bmx-SK expression in muscle of Bmx-SK-Tg mice (Tg lines 4 and 6). Mouse lower hind limb was collected and homogenized. Bmx-SK was detected by Western blotting with anti-Flag antibody. C57BL/6 muscle tissue (WT) was used as a negative control. $\beta$-Tubulin was used for protein normalization. (E) Relative expression level of Bmx-SK and endogenous Bmx. Expression of both transgenic and endogenous Bmx in hind limb was determined by Western blotting with anti-Bmx. Bmx, Bmx-SK, and nonspecific bands (NS) are indicated. (F) Bmx-SK expression in muscle capillaries of Bmx-SK-Tg mice. Mouse lower hind limb was collected as frozen sections, and Bmx-SK and EC markers were detected by immunohistochemistry with anti-Bmx and anti-CD31 antibodies, respectively. Bmx-SK-positive capillaries are indicated by arrows. (G) C57BL/6 (WT) and Bmx-KO (KO) muscle tissues were used as negative and a positive controls, respectively.

$B m x-K O$ and $B m x-S K-T g$ mice. The upregulation of Bmx in ischemic hind limb prompted us to reason that Bmx might play a critical role in ischemia-induced tissue repair. The availability of Bmx-KO mice that are developmentally "normal" permits us test the role of Bmx in postnatal hind limb angiogenesis and remodeling, despite its relative lack of importance during embryonic vascular development (24). Because Bmx was primarily expressed in vascular endothelium and bone marrow, and can be induced in vascular endothelium including capillaries, we have also generated endothelium-specific Bmx-transgenic mice to test our hypothesis. The transgenic mice express, via use of a Tie-2 enhancer/promoter, a constitutively active form of Bmx containing the SH2 and the kinase domains (Bmx-SK) (Figure 2A). We chose the longer Tie-2 enhancer fragment $(15-\mathrm{kb})$ rather than the short one $(1.6-\mathrm{kb})$ because it provides uniform protein expression in both the bone marrow and vascular endothelium, especially in adult tissues (26). We obtained 2 founders as determined by PCR genotyping (Figure 2B) and successfully bred them in the C57BL/ 6 background for 10 generations. Bmx-SK mRNA from the 2 lines (Tg lines 4 and 6) was highly expressed in vascularized tissues including bone marrow, aorta, brain, heart, and hind limb muscle as determined by qRT-PCR (Figure 2C). Bmx-SK protein was expressed in hind limb muscle tissue of Bmx-SK-Tg mice (both Tg line 4 and line 6) but not of C57BL/6 mice, as determined by Western blotting with anti-Flag antibody (Figure 2D). Both lines of Bmx-SK-Tg mice showed normal development, growth, and breeding behavior. We chose transgene line 4 for further analyses. We also determined the relative expression level of the Bmx-SK transgene and endogenous Bmx by Western blotting with anti-Bmx antibody. The level of Bmx-SK transgene was 4-fold higher than that of the endogenous Bmx protein, which was not altered by the transgene (Figure 2E). Furthermore, Bmx-SK was specifically detected in vessels of hind limb as determined by immunohistochemistry with anti-Bmx antibody and anti-CD31, 
A Mouse model of critical limb ischemia

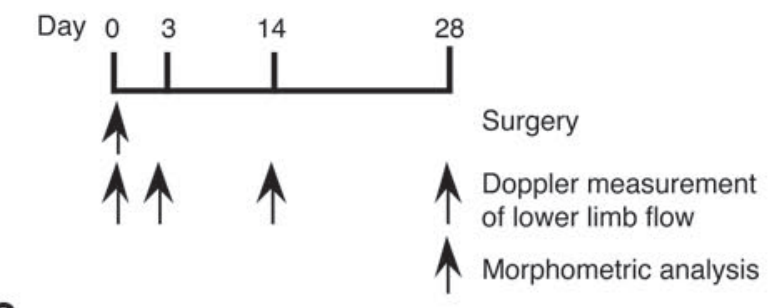

C
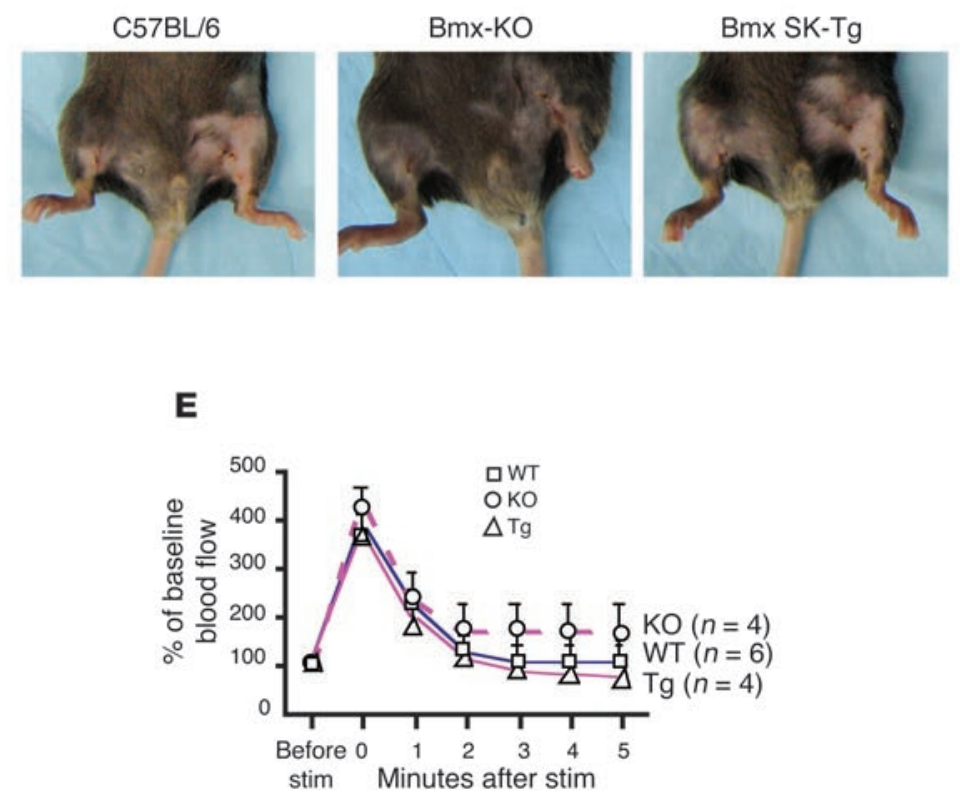
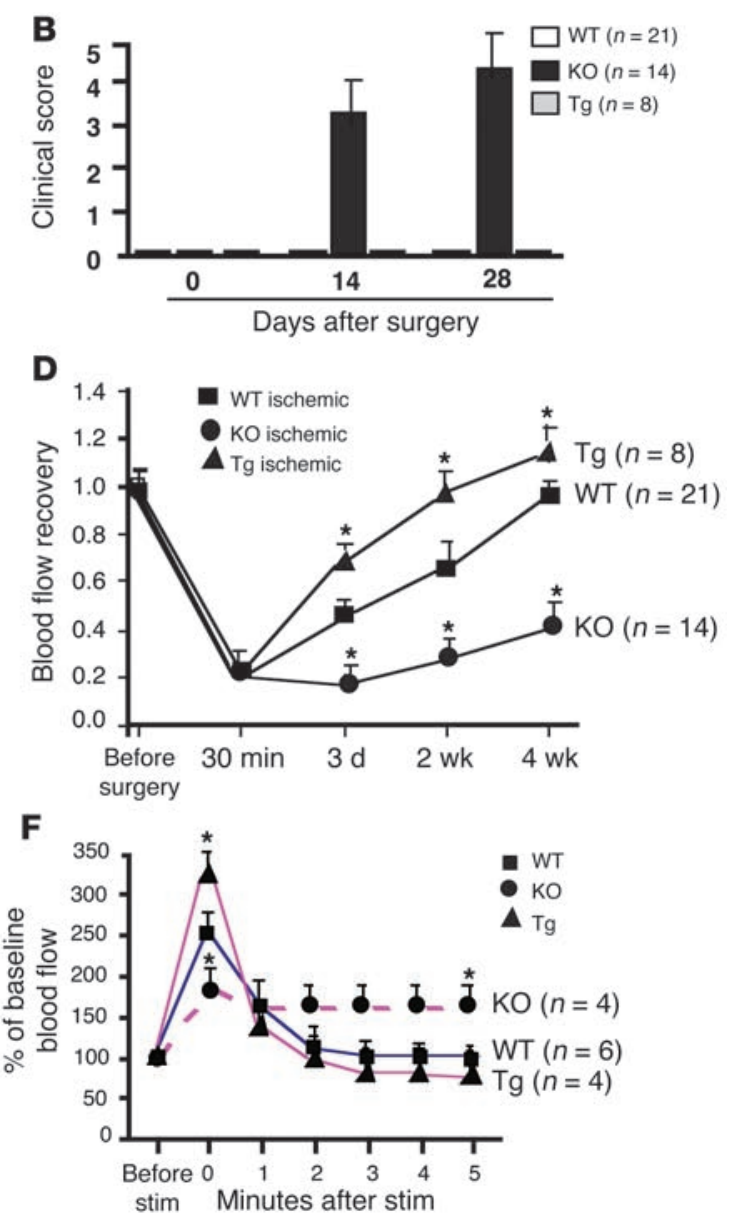

Figure 3

Critical roles of Bmx in the recovery of hind limb perfusion after injury. (A) Hind limb ischemia was induced. Blood flow of ischemic (left) and nonischemic (right) limb were measured on gastrocnemius muscle at 30 minutes, 3 days, 2 weeks, and 4 weeks after surgery using the PeriFlux system with a Laser Doppler Perfusion module unit (LDPU). Tissues were harvested on day 28 for immunohistochemistry. In Bmx-KO mice, clinical score indicated a severe phenotype (B), leading to necrosis of limb (C). In contrast, Bmx-SK-Tg mice, like C57BL/6 mice, recovered completely after 4 weeks. (D) Bmx-SK-Tg mice showed augmented, whereas Bmx-KO mice showed reduced, recovery of limb perfusion compared with normal C57BL/6 mice (ratios of perfusion in nonischemic [left] limb to that in ischemic [right] limb are shown). (E and F) Bmx-SK-Tg mice show enhanced, while Bmx-KO mice show abnormal, postcontraction hyperemia. Adductor muscle groups of mice from before surgery (E) and 2 weeks after surgery $(\mathbf{F})$ were electrostimulated, and increase in gastrocnemius blood flow was recorded. Both baseline and stimulated lower leg perfusion were measured as an index of the maximal vasodilatory capacity. Data are mean $\pm \mathrm{SEM} ;{ }^{*} P<0.05$. stim, stimulation.

respectively (Figure 2F). In control experiments, a basal level of $\mathrm{Bmx}$ was detected in the vasculature of $\mathrm{C} 57 \mathrm{BL} / 6$ mice but not of Bmx-KO mice (Figure 2G).

$B m x-K O$ blunts, whereas Bmx-SK-Tg augments, perfusion recovery in ischemic hind limbs. C57BL/6, Bmx-KO, and Bmx-SK-Tg male mice were subjected to femoral artery ligation and analysis at different time points as diagrammed in Figure 3A. On day 14-28 after surgery, Bmx-KO mice showed a severe phenotype compared with C57BL/ 6 and Bmx-SK-Tg mice (Figure 3B) based on the clinical scoring system that we described recently (27). Ten of $14 \mathrm{Bmx}-\mathrm{KO}$ mice had severe necrosis of the feet (Figure 3C). To precisely determine functional defects in $\mathrm{Bmx}-\mathrm{KO}$ mice, blood flow was measured and ischemic and nonischemic limb perfusion were measured before and directly after surgery and then 3 days, 2 weeks, and 4 weeks after surgery using a deep-penetrating laser probe placed onto the gastrocnemius. Before surgery, the ratio of left leg to right leg gastrocnemius blood flow is 1 . After surgery, flow dropped by
$80 \%$ and returned to a ratio of 1 over 4 weeks in C57BL/6 mice. There was a marked impairment in gastrocnemius blood flow in $\mathrm{Bmx}-\mathrm{KO}$ mice, suggesting that the impairment in blood flow in $\mathrm{Bmx}-\mathrm{KO}$ is associated with a marked increase in clinical severity (Figure 3D). In contrast, both lines of Bmx-SK-Tg mice $(n=8$ for each line) showed augmented recovery of hind limb perfusion, and flow returned to normal in 2 weeks (Figure 3D, shown for Tg line 4). We focused $\mathrm{Tg}$ line 4 for further analyses.

To further define the functional defects in Bmx-KO mice, we examined skeletal muscle contraction-stimulated hyperemia in the gastrocnemius muscle in C57BL/6, Bmx-SK-Tg, and Bmx-KO mice at baseline and after ischemia, as we described recently for eNOS-KO mice (28). As seen in the typical traces in Figure 3E, electrical stimulation of the adductor muscle groups in the upper legs of $\mathrm{C} 57 \mathrm{BL} / 6, \mathrm{Bmx}-\mathrm{SK}-\mathrm{Tg}$, or Bmx-KO mice resulted in a marked increase in peak blood flow measured in the gastrocnemius muscle group of all strains (compare the prestimulation level 
A
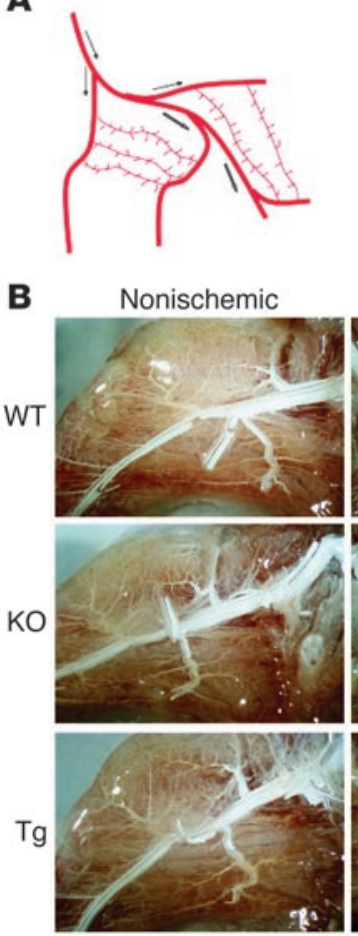

C

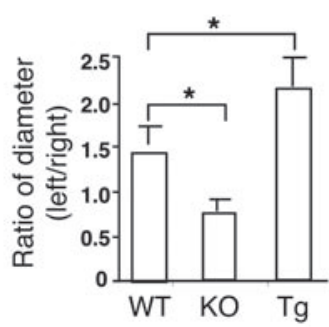

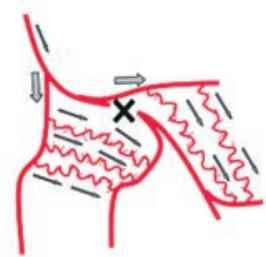

\section{D \\ WT}

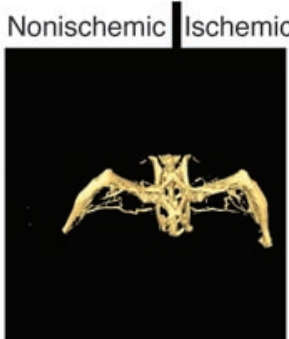

Ischemic

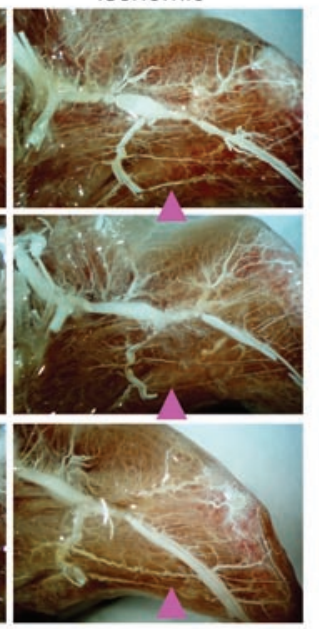

$\mathrm{KO}$

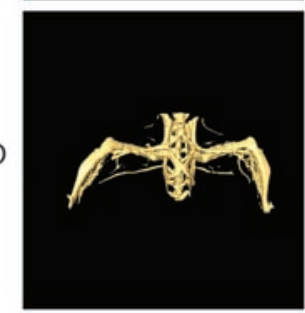

$\operatorname{Tg}$

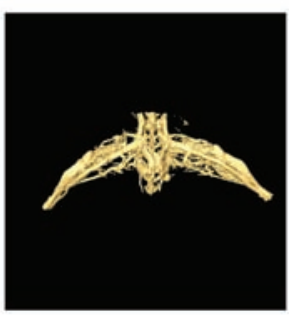

E

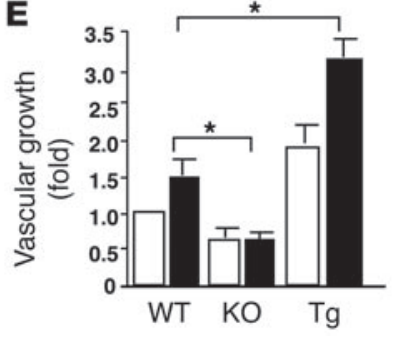

\section{Figure 4}

Critical roles of $\mathrm{Bmx}$ in ischemia-induced arteriogenesis. (A) Diagram of blood flow changes after occlusion of the femoral artery in the hind limb. After occlusion of the femoral artery (X, right panel), blood flow (arrows) follows the gradient between high pressures proximal to the occlusion site and very low distal pressures. Preexisting collateral arterioles are recruited. (B) Arteriogenesis was determined by MICROFIL casting analyses. Four weeks after femoral ligation, mice were anesthetized and subjected to MICROFIL perfusion. Collateral artery growth is indicated by arrows. (C) Arterial growth was quantitated as ratio of diameters (left artery/right artery), and $n=10$ for each strain. ${ }^{*} P<0.05$. (D) microCT analyses. MICROFIL casting samples were subjected to microCT analyses as described in Methods. (E) Vascular growth was quantitated as ratio of vascular density (left leg/right leg). $n=10$ for each strain. ${ }^{*} P<0.05$. with the peak response upon stimulation [time 0]). The return to baseline flow was slightly delayed in Bmx-KO mice compared with C57BL/ 6 or Bmx-SK-Tg mice. Next, we measured the same physiological response in $\mathrm{C} 57 \mathrm{BL} / 6, \mathrm{Bmx}-\mathrm{SK}-\mathrm{Tg}$, and $\mathrm{Bmx}-\mathrm{KO}$ mice at 2 weeks after ischemia. Electrical stimulation of the adductor muscle groups in the upper leg of Bmx-SK-Tg mice resulted in an increase in peak blood flow in the gastrocnemius muscle group similar to that occurring before ischemia (Figure 3, F versus E). C57BL/ 6 mice showed a peak response that was $70 \%$ of that before ischemia. However, Bmx-KO mice showed a markedly diminished peak response (approximately 30\%), and the return was significantly delayed compared with that before ischemia. These data show that Bmx-SK-Tg mice have enhanced, whereas Bmx-KO have reduced, hyperemic responses after ischemia.

Postischemic arteriogenesis is enhanced in Bmx-SK-Tg mice but is impaired in Bmx-KO mice. Enhanced clinical recovery and limb perfusion could be due to increased arteriogenesis from existing vessels of the upper limb, increased neovascularization/vessel maturation in the lower limb, and increased mobilization of endothelial cell progenitors. We first examined ischemia-initiated arteriogenesis in C57BL/6, Bmx-KO, and Bmx-SK-Tg mice by MICROFIL casting to visualize vascular architecture. Time-course studies in C57BL/6 mice suggested that collateral artery growth and tissue perfusion in the ischemic hind limb peaked at 4 weeks after ischemia (Supplemental Figure 2). We then compared the collateral growth in $\mathrm{C} 57 \mathrm{BL} / 6, \mathrm{Bmx}-\mathrm{KO}$, and Bmx-SK-Tg mice. As diagrammed in Figure $4 \mathrm{~A}$ and shown in Figure 4B, there was an enlargement of collaterals compared with contralaterals in C57BL/6 (WT) mice after 4 weeks of ischemia. Bmx-SK-Tg mice showed enhanced arterialization as determined by the ratio of diameters in the left arteries to those in the right arteries (Figure 4B; quantification in Figure 4C). In contrast, $\mathrm{Bmx}-\mathrm{KO}$ mice showed a complete lack of enlargement of the collaterals (Figure 4, B and C).

MICROFIL casting primarily visualized the surface vasculature of hind limbs. To better quantify vascular density in the upper and lower limbs of mice, we further performed 3D microCT with these MICROFIL-casting samples. The vasculature was volume rendered using the 3D semiautomated image analysis described in Methods. Both vasculature and the bone were displayed in 3D (Figure 4D). The bone was manually wiped, and vasculature was quantified by image analysis (Methods). Consistent with the results of image analysis of the surface vasculature (Figure 4, B and $\mathrm{C}$ ), vascular density was reduced in $\mathrm{Bmx}-\mathrm{KO}$ mice, whereas it was significantly increased in Bmx-SK-Tg mice compared with WT mice (Figure 4E). These data document a critical role of $\mathrm{Bmx}$ in ischemia-mediated arteriogenesis. 
A
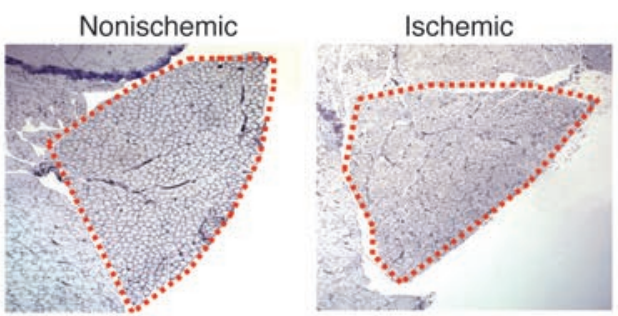

B

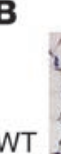
Nonischemic

WT

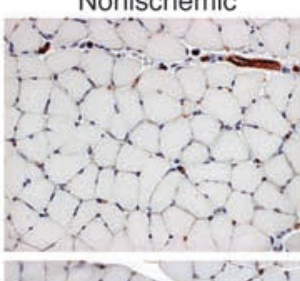

$\mathrm{KO}$
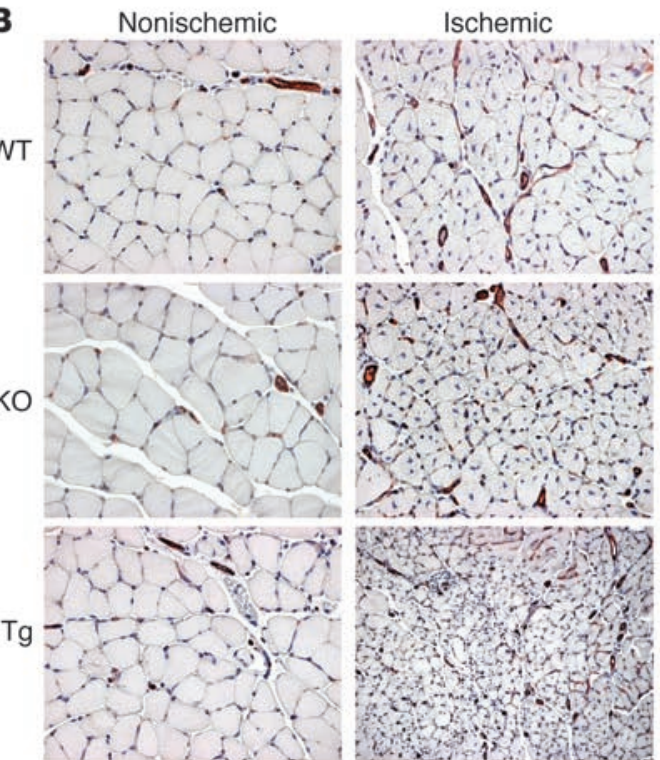
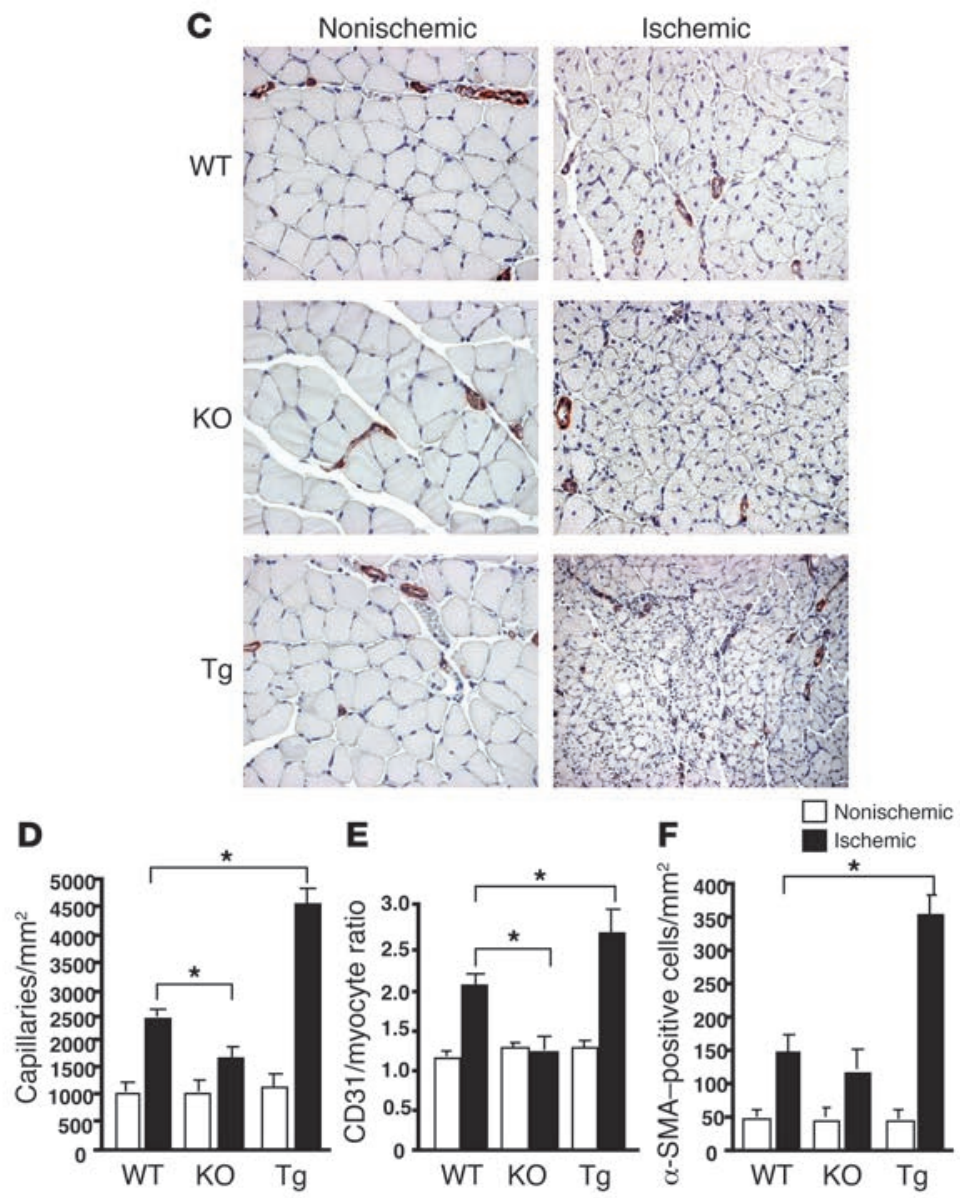

Figure 5

Critical roles of $\mathrm{Bmx}$ in ischemia-induced neovascularization and pericyte recruitment. (A) Characterization of ischemia-responsive regions. The region of the gastrocnemius muscles outlined in red was the most responsive to ischemia and was selected for further analyses. The region was immunostained with CD31 (an EC marker) (B) and $\alpha$-SMA (a smooth muscle/pericyte marker) (C) to determine capillary density and pericyte recruitment, respectively. Representative sections from nonischemic and ischemic groups of C57BL/6, Bmx-KO, and Bmx-SK-Tg mice on day 3 after ischemia are shown. (D-F) Quantitation of capillaries (number $/ \mathrm{mm}^{2}$ muscle area), ratio of CD31-positive to total myocytes, and $\alpha$-SMA-positive staining (number $/ \mathrm{mm}^{2}$ muscle area). Data are mean \pm SEM from 10 fields per section (3 sections/mouse and $n=4$ for each strain). ${ }^{*} P<0.05$.

Ischemia-induced angiogenesis and vessel maturation are enhanced in $B m x-S K-T g$ mice but are impaired in B $m x-K O$ mice. Secondary to upper limb ischemia, shear stress-dependent changes in blood flow will promote capillary angiogenesis in the lower leg. We first characterized the ischemia-responsive regions in the lower limb, and we found that a triangular area of gastrocnemius muscle was most responsive to ischemia when measured for increased capillary formation, macrophage infiltration, and myocyte proliferation (Figure 5A). Thus, we focused on this region to determine ischemia-induced angiogenesis. After 4 weeks of ischemia, there was an increase in the number of CD31-positive capillaries surrounding the skeletal muscle fibers in C57BL/6 mice (Figure 5B; quantification of the number of capillaries and capillary/fiber ratio in Figure 5, D and E, respectively). Stable angiogenesis is believed to occur contemporaneously with pericyte recruitment (vessel maturation) mediated by PDGF and/or angiopoietin-1. In order to examine the recruitment of pericytes to angiogenic capillary sprouts after ischemia, thin serial sections were immunostained with anti-CD31 or anti- $\alpha$-SMA antibody. The number of $\alpha$-SMApositive capillaries was also increased in C57BL/ 6 after 4 weeks of ischemia (Figure 5C). Importantly, the numbers of CD31-positive capillaries surrounding the skeletal muscle fibers (neovascularization) and $\alpha$-SMA-positive SMCs (pericyte recruitment) were significantly increased in Bmx-SK-Tg mice but reduced in $\mathrm{Bmx}-\mathrm{KO}$ compared with C57BL/6 mice secondary to ischemia (Figure 5, $B$ and $C$, respectively). Results were quantified as capillary number per millimeter squared, capillary/fiber ratio, or $\alpha$-SMA-positive vessels per millimeter squared in Figure 5, D-F.

$B m x$ is critical for ischemia-induced TNFR2 and VEGFR2 angiogenic signaling. To determine the molecular mechanism by which Bmx regulates ischemia-induced arteriogenesis/angiogenesis as observed above, we examined the recruitment of inflammatory/immune cells (primarily macrophages and lymphocytes), which have been shown to be critical for ischemia-mediated arteriogenesis/angiogenesis $(4,29)$. Infiltration of macrophages and lymphocytes to ischemic hind limb was determined by immunostaining with the anti-F4/80 and anti-CD3 antibodies, respectively. No macrophages were detected in nonischemic limb. Ischemia induced a drastic increase in infiltration of macrophages, which peaked at day 3 after surgery $\left(\sim 500\right.$ macrophages $\left./ \mathrm{mm}^{2}\right)$ and declined by day 7 in 

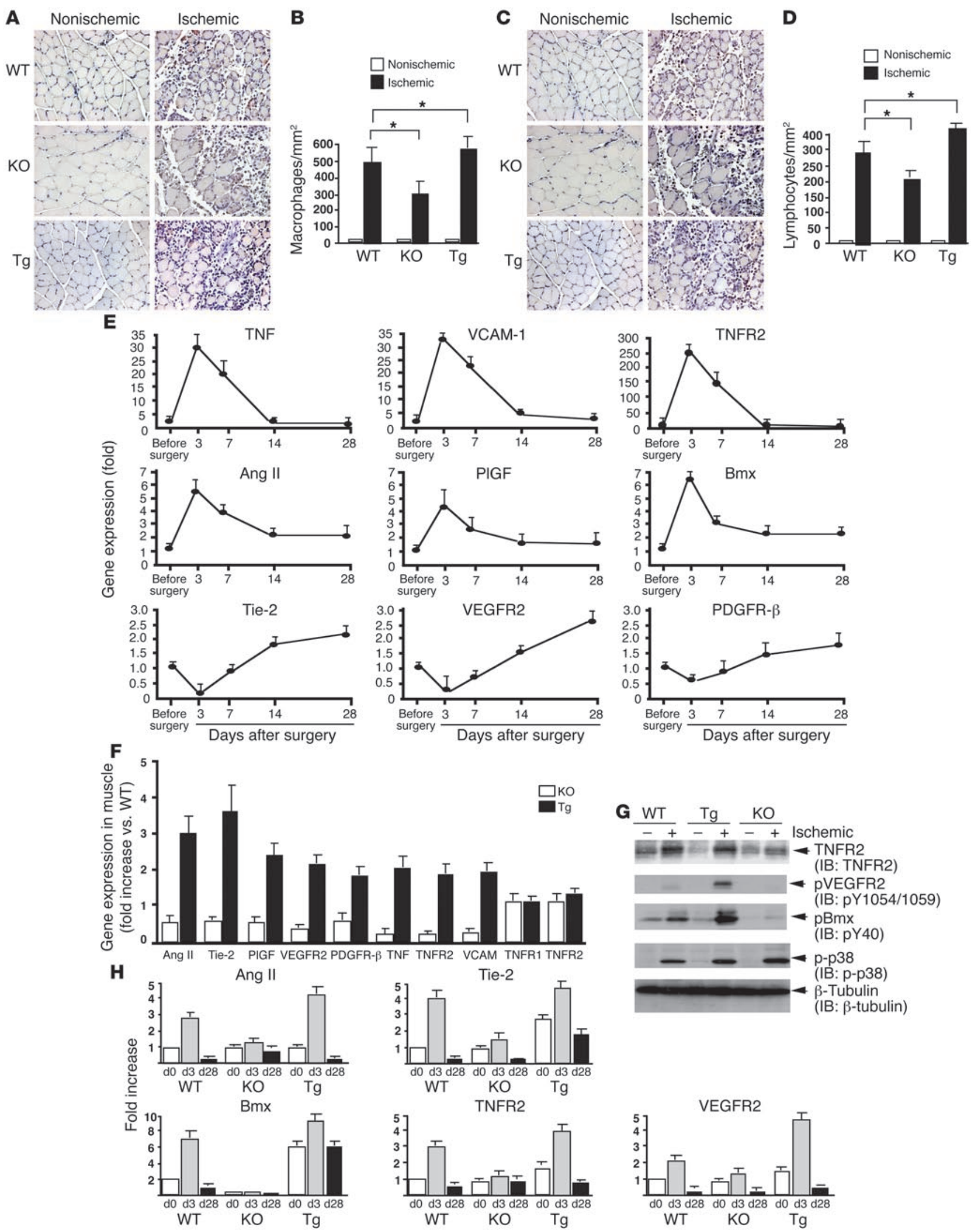


\section{Figure 6}

Characterization of infiltrated immune cells, gene expression, and Bmx/TNFR2/VEGFR2 signaling in response to ischemia. (A-D) Recruitment of macrophages/lymphocytes in response to ischemia as determined by anti-F4/80 and anti-CD3 antibodies, respectively. Representative images of nonischemic and ischemic hind limbs in C57BL/6 mice on day 3 are shown for macrophages (A) and for lymphocytes (C). F4/80- and CD3-positive cells were counted as number infiltrating per millimeter squared muscle area (B and $\mathbf{D})$. (E) The expression of proinflammatory and proangiogenic genes was assessed by qRT-PCR in the gastrocnemius $0,3,7,14$, and 28 days after ischemia in C57BL/6 mice. (F) Ischemia-induced gene expression in Bmx-KO and Bmx-SK-Tg mice on day 3 (early-responsive genes) or day 28 (late-responsive genes, VEGFR2, Tie-2, and PDGFR- $\beta$ ) after ischemia was determined by qRT-PCR. Data represent the fold increases of each gene, with expression in C57BL/6 mice taken as 1.0 . $n=3$ for each strain per time point. (G) Bmx is critical for ischemia-induced expression/activation of TNFR2 and VEGFR2. Day 7 postsurgery samples were used for protein analyses. Expression of TNFR2 and phosphorylation of Bmx (pY40), VEGFR2 (pY1154/1159), and p38 were determined by Western blotting with respective antibodies. $\beta$-Tubulin protein was used for normalization. $(\mathbf{H})$ Ischemia-induced gene expression in bone marrow of WT, Bmx-KO, and Bmx-SK-Tg mice on day 3 and day 28 after ischemia was determined by qRT-PCR. Data represent the fold increases of each gene, with expression in WT mice without ligation taken as 1.0. $n=3$ for each strain per time point.

C57BL/6 mice (Supplemental Figure 3A). Similar kinetics were observed for lymphocyte infiltration (Supplemental Figure 3B). We then determined infiltration of macrophages/lymphocytes in Bmx-KO and Bmx-SK-Tg mice on day 3. Results showed a significant reduction in macrophage/ $\mathrm{T}$ cell infiltration in $\mathrm{Bmx}-\mathrm{KO}$ mice and a significant increase in Bmx-SK-Tg mice compared with C57BL/6 mice (Figure 6, A and B, for macrophages; and Figure 6, $\mathrm{C}$ and $\mathrm{D}$, for $\mathrm{T}$ cells). These data suggest a role of $\mathrm{Bmx}$ in the recruitment of infiltrated cells, consistent with its expression in hematopoietic cells (6-13).

Next, we examined gene expression profiles of several growth factors and their cognate receptors that may contribute to ischemia-induced arteriogenesis/angiogenesis. Gene expression of angiogenic factors and proinflammatory cytokines as well as their cognate receptors was determined by qRT-PCR. The levels of gene expression were compared in ischemic contralateral and nonischemic gastrocnemius muscle groups. Data represent the fold increase of each gene with the value for the nonischemic hind limb of C57BL/6 mice taken as 1.0. Ischemia rapidly induced the expression of proinflammatory genes (TNF, TNFR2, and VCAM-1) and angiogenic molecules (Ang I, Ang II, placental growth fac-

\section{Figure 7}

Bmx in bone marrow-derived cells contributes to an early phase of ischemia-induced arteriogenesis/angiogenesis. (A) Characterization of BMT. C57BL/6 mice were subjected to lethal irradiation followed by BMT by receiving bone marrow cells from WT (WT to WT), Bmx-KO mice (KO to WT), or Bmx-SK-Tg mice (Tg to WT). Success of BMT was controlled by genotyping of Bmx gene 6 weeks after BMT. The mice were then subjected to femoral artery ligation as described in Methods. Four weeks after ischemia, bone marrow was harvested, and expression of Bmx and Bmx-SK in BM was determined by Western blotting with anti-Bmx antibody. Bone marrow from WT and Bmx-SK-Tg mice without BMT/ligation was used as control. Expression of $\mathrm{Bmx}$ and $\mathrm{Bmx}-\mathrm{SK}$ is indicated. Anti- $\beta$-tubulin antibody was used for normalization. (B) Pedal blood flow recovery after femoral artery ligation in C57BL/6 (WT Ctrl) versus mice that received bone marrow from WT (BMT of WT to WT), Bmx-KO mice (BMT of KO to WT), or Bmx-SK-Tg mice (BMT of Tg to WT). BMT was performed, and pedal blood flow recovery on indicated days after femoral artery ligation was assessed by laser Doppler imaging. ${ }^{*} P<0.05$. (C) BMT of WT to Bmx-KO mice. Bmx-KO mice were subjected to lethal irradiation followed by BMT by receiving bone marrow cells from WT mice (WT to KO). Successful BMT was controlled by genotyping of the Bmx gene 6 weeks after BMT. The mice were then subjected to femoral artery ligation as described in Methods. WT and Bmx-KO mice without BMT were used as controls. Pedal blood flow recovery after femoral artery ligation was assessed by laser Doppler imaging. $n=10$ for each strain. ${ }^{\star} P<0.05$. tor [PlGF], and Bmx). Expression of these early-responsive genes peaked at day 3 and declined by day 14. The TNFR2 gene showed the most drastic induction by ischemia (up to 250-fold) (Figure 6E). However, the cognate receptors VEGFR2, Tie-2, and PDGFR- $\beta$, primarily expressed on ECs and/or SMCs/pericytes, decreased their expression on day 3 but increased their expression on day 14 and day 28, consistent with the kinetics of flow recovery, in the ischemic hind limb, probably reflecting the pattern of vessel growth and maturation. In contrast, expression of several genes, including TNFR1 and TNFR-associated factor-2 (TRAF2), was not significantly altered (data not shown). We then compared the expression of these genes in Bmx-KO and Bmx-SK-Tg mice. The

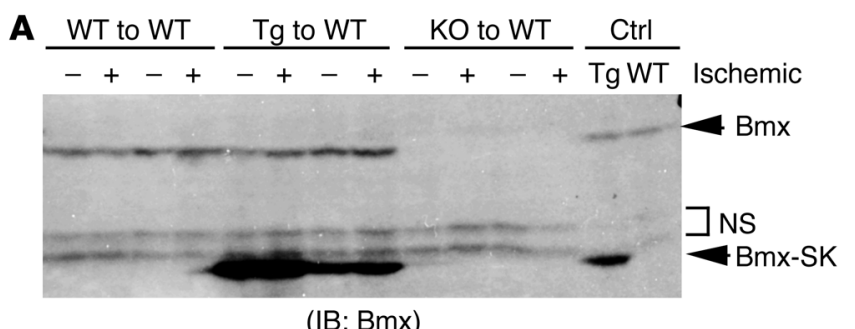

(IB: $\mathrm{Bmx})$

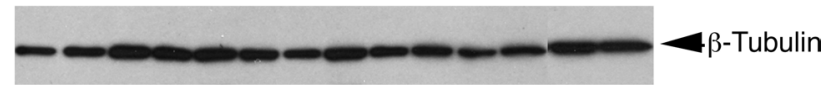

(IB: $\beta$-tubulin)

B

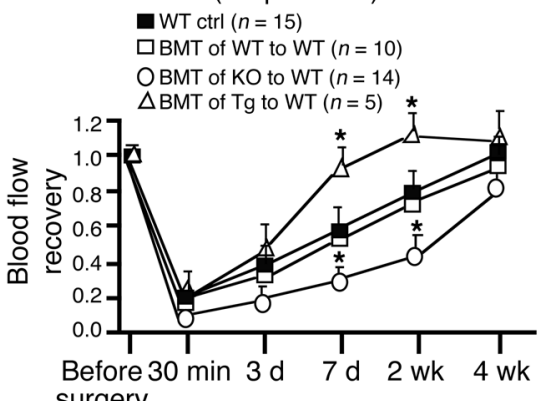

Before 30
surgery

C

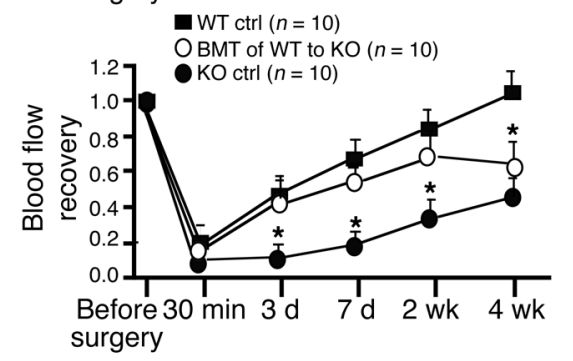




\section{Table 1}

Complete blood counts in WT, Bmx-KO, and Bmx-SK-Tg mice

\begin{tabular}{|c|c|c|c|c|c|c|c|c|}
\hline & $\begin{array}{l}\text { Hemoglobin } \\
(\mathrm{g} / \mathrm{dl})\end{array}$ & $\begin{array}{l}\text { Hematocrit } \\
(\%)\end{array}$ & $\begin{array}{c}\text { rbc } \\
\left(10^{6} / \mu \mathrm{l}\right)\end{array}$ & $\begin{array}{c}\text { Platelets } \\
\left(10^{6} / \mu \mathrm{l}\right)\end{array}$ & $\begin{array}{c}\text { wbc } \\
\left(10^{3} / \mu l\right)\end{array}$ & $\begin{array}{l}\text { Neutrophils } \\
\left(10^{3} / \mu \mathrm{l}\right)\end{array}$ & $\begin{array}{l}\text { Lymphocytes } \\
\left(10^{3} / \mu \mathrm{I}\right)\end{array}$ & $\begin{array}{c}\text { Monocytes } \\
\left(10^{3} / \mu \mathrm{l}\right)\end{array}$ \\
\hline \multicolumn{9}{|l|}{ Basal } \\
\hline WT & 13.9 & 37 & 8.63 & 1.2 & 3.6 & 0.88 & 2.1 & 0.044 \\
\hline $\mathrm{Tg}$ & 13 & 40.5 & 9 & 1.3 & 3.7 & 0.79 & 2.2 & 0.046 \\
\hline KO & 13.5 & 38 & 8.5 & 0.9 & 3.8 & 0.92 & 2.0 & 0.049 \\
\hline \multicolumn{9}{|l|}{ BMT ctrl } \\
\hline WT to WT & 14.9 & 40.8 & 10.3 & 1.2 & 3.5 & 0.86 & 1.8 & 0.049 \\
\hline $\mathrm{Tg}$ to WT & 13.6 & 42 & 9.4 & 1.1 & 3.6 & 0.98 & 2.3 & 0.057 \\
\hline KO to WT & 13.5 & 40.5 & 8.9 & 0.99 & 3.6 & 0.96 & 2.1 & 0.052 \\
\hline \multicolumn{9}{|c|}{ BMT, ischemic, day 3} \\
\hline WT to WT & 13.1 & 41 & 8.2 & 0.96 & 3.6 & 0.9 & 2.3 & $0.06^{A}$ \\
\hline Tg to WT & 13.3 & 42.2 & 8.8 & 0.97 & 3.7 & 1 & 2.4 & $0.065^{\mathrm{A}}$ \\
\hline KO to WT & 12.5 & 38.8 & 8.6 & 0.96 & 3.9 & 1.2 & 2.5 & $0.066^{A}$ \\
\hline \multicolumn{9}{|c|}{ BMT, ischemic, day 28} \\
\hline WT to WT & 11 & 35.5 & 7.8 & 1.1 & $6.4^{\mathrm{B}}$ & $1.6^{\mathrm{B}}$ & $4.5^{\mathrm{B}}$ & $0.063^{\mathrm{A}}$ \\
\hline $\mathrm{Tg}$ to WT & 12 & 38.2 & 8.3 & 1.1 & $5.2^{\mathrm{B}}$ & $1.3^{\mathrm{B}}$ & $3.8^{\mathrm{B}}$ & $0.068^{\mathrm{A}}$ \\
\hline KO to WT & 12.5 & 39.9 & 7.6 & 0.95 & $6.2^{\mathrm{B}}$ & $1.9^{\mathrm{B}}$ & $3.9^{\mathrm{B}}$ & $0.075^{\mathrm{A}}$ \\
\hline
\end{tabular}

Peripheral blood from WT, Bmx-KO, and Bmx-SK Tg mice was collected before (Basal) and after BMT (BMT ctrl) and on indicated days after ischemia. Alncreases in number of monocytes on day 3 after ischemia. ${ }^{B}$ Increases in numbers of total wbc and individual cell types.

results showed that expression of these angiogenic and proinflammatory genes was reduced by 2 - to 5 -fold in $\mathrm{Bmx}-\mathrm{KO}$ mice but increased by 1.8- to 3.5-fold in Bmx-SK-Tg mice compared with WT mice (Figure 6F). Finally, we determined the effects of Bmx on TNFR2 and VEGFR2 signaling, which have been shown to be critical for Bmx activation $(9,10)$. Results showed that expression of TNFR 2 and activation of VEGFR 2 and Bmx on day 7 were significantly induced by ischemia. Interestingly, these ischemic responses were enhanced in Bmx-SK-Tg mice, whereas they were blunted in Bmx-KO mice (Figure 6G). In control experiments, Bmx had no effects on ischemic/inflammation-induced activation of stressactivated kinase p38 (Figure 6G), suggesting that Bmx had specific effects on TNFR2 and VEGFR2.

We also analyzed gene expression in bone marrow by qRT-PCR. Gene expression of proinflammatory molecules (TNF, VCAM-1, and TNFR1) was not altered in response to ischemia. However, several critical angiogenic molecules, including Ang II, Tie-2, Bmx, TNFR2, and VEGFR2 were significantly upregulated on day 3 , followed by a decline on day 28 after ischemia (Figure $6 \mathrm{H}$ ). More importantly, gene expression of these angiogenic molecules in bone marrow was Bmx dependent, as observed in the hind limb (Figure $6 \mathrm{H}$ ). Taken together, these data suggest that $\mathrm{Bmx}$ regulates gene expression of angiogenic and proinflammatory genes in hind limb and bone marrow.

Bmx in bone marrow-derived cells contributes to an early phase of ischemia-induced arteriogenesis/angiogenesis. Mobilization of immune cells and EPCs from bone marrow to peripheral blood and injury sites has been proposed to account for the function of bone marrow cells (30-33). Bmx was initially identified as a bone marrow tyrosine kinase (6), which led us to determine the role of bone morrow-derived cells in ischemia-induced angiogenesis. To this end, we performed bone marrow transplantation (BMT) experiments using C57BL/6 mice as recipients. First, C57BL/6 (WT) mice received lethal irradiation, followed by transplantation of bone morrow cells from WT, Bmx-KO, or Bmx-SK-Tg mice. Six weeks after BMT, the mice were subjected to femoral artery ligation. Success of BMT was verified by genotyping of blood samples with Bmx-KO- or Bmx-SK-specific primers (data not shown) and by Western blotting of bone marrow samples with anti-Bmx antibody (Figure 7A). Furthermore, we performed complete blood tests using peripheral blood from WT, Bmx-KO, and Bmx-SK-Tg mice before and after BMT. Results indicated that bone marrow in BMT mice was completely reconstituted by 6 weeks after BMT according to hemoglobin levels, hematocrit, and numbers of red blood cells, platelets, and total white blood cells, as well as individual cell types such as neutrophils, lymphocytes, and monocytes (compare Basal and BMT ctrl in Table 1). Blood cell counting was also performed on day 3 and day 28 after ischemia. Results showed that the number of monocytes was increased on day 3 after ischemia, and increases in the numbers of total white blood cells as well as individual cell types (monocytes, neutrophils, and lymphocytes) were observed on day 28 (Table 1). However, no significant differences among the 3 strains were detected.

WT mice without BMT (WT ctrl) were used as control for ligation and flow measurements, and no significant differences in blood flow recovery were observed between WT ctrl and WT BMT groups (Figure 7B). Importantly, blood flow recovery in transplanted mice was similar to that in the donor strain. Specifically, blood flow recovery was significantly impaired in BMT of Bmx-KO to WT mice, whereas it was dramatically increased in BMT of Bmx-SK-Tg to WT mice compared with WT ctrl on day 7 and day 14 (Figure 7B). Interestingly, no statistically significant differences were observed on day 28 among these groups (Figure 7B), suggesting that $\mathrm{Bmx}$ in bone marrow-derived cells might contribute to the early phase of ischemiainduced flow recovery. To further test this possibility, we performed BMT of WT to Bmx-KO mice. Success of BMT was verified by genotyping of blood samples with Bmx-specific primers (data not shown) and by Western blotting of bone marrow samples with anti-Bmx antibody (Supplemental Figure 4). WT and Bmx-KO mice without 
BMT were used as controls (WT Ctrl and KO Ctrl). Results showed that blood flow recovery in transplanted mice (WT to KO) was similar to that in WT Ctrl in the first 2 weeks. However, blood flow recovery was significantly impaired in BMT of WT to Bmx-KO mice on day 28 and was close to that observed in $\mathrm{KO}$ ctrl mice (Figure 7C; also see Figure 3). Taken together, the results of our study suggest that $\mathrm{Bmx}$ in bone marrow-derived cells is critical for the early phase of ischemia-induced tissue repair, whereas $\mathrm{Bmx}$ in intrinsic vasculature contributes to the later phase of arteriogenesis/angiogenesis.

\section{Discussion}

The most important finding of this study is that $\mathrm{Bmx}$, a non-receptor tyrosine kinase expressed in bone marrow and endothelium, plays a critical role in mediating ischemia-induced arteriogenesis/ angiogenesis in vivo. This conclusion is based on the functional analyses from both genetically deficient $\mathrm{Bmx}$ mice and transgenic mice expressing a constitutively active form of $\mathrm{Bmx}(\mathrm{Bmx}-\mathrm{SK}-\mathrm{Tg})$ in bone marrow and endothelial cells. Specifically, in response to ischemia, Bmx-KO mice showed marked deficits, whereas Bmx-SK-Tg mice had enhanced clinical recovery, limb perfusion, and ischemic reserve capacity compared with C57BL/6 mice. MICROFIL casting, 3D microCT analyses, and histological analyses showed that $\mathrm{Bmx}-\mathrm{KO}$ mice have decreased, while Bmx-SK-Tg mice have increased, ischemia-initiated arteriogenesis in the upper limb and capillary formation and vessel maturation in the lower limb. Furthermore, Bmx and Bmx-SK were detected in bone marrow, and results of BMT studies showed that Bmx in bone marrow-derived cells plays a critical role in the early phase of ischemic tissue remodeling. Further BMT studies demonstrated that $\mathrm{Bmx}$ in intrinsic vasculature contributes to the later phase of arteriogenesis/angiogenesis. Our studies provide the first data to our knowledge on Bmx function in bone marrow and vasculature, where $\mathrm{Bmx}$ is primarily expressed in vivo (24).

Although the Bmx-deficient mice are normal in embryonic vasculogenesis (24), our study here demonstrates that Bmx plays critical roles in ischemia-mediated arteriogenesis and angiogenesis in adult tissues. It has been demonstrated that several genes play important roles in pathological but not physiological angiogenesis. The availability of KO mice that are developmentally "normal" permits the testing of these genes required for postnatal hind limb angiogenesis and remodeling, despite their relative lack of importance during vasculogenesis. The VEGF homolog PlGF is one example. It has been demonstrated by P. Carmeliet's group that embryonic angiogenesis in mice was not affected by the deficiency of PlGF (Pgf $-/$ ). However, loss of PlGF impaired angiogenesis, plasma extravasation, and collateral growth during ischemia, inflammation, wound healing, and cancer (34). Other examples of impaired ischemic limb arteriogenesis and angiogenesis can be found in mice deficient in IL-10 (35), the Ang II type 1 receptor (36), MMP-9 (37), adiponectin (38), caveolin-1 (39), and, according to some but not all studies, the receptor for monocyte chemoattractant protein 1 (MCP1), CC chemokine receptor 2 (CCR2) $(40,41)$. We and others have shown that eNOS is also important for ischemia-induced arteriogenesis/angiogenesis and blood flow recovery $(28,32,42)$. Our study here indicates that $\mathrm{Bmx}$ is another member in this group of genes. The underlying mechanisms by which these genes are of functional importance in pathological but not physiological angiogenesis are not understood. One possible explanation is that these genes are expressed or activated or associated with downstream targets only in response to pathological stimuli. Indeed, we observed that Bmx expression and activity are strongly upregulated in vasculature in response to ischemia. Consistent with this notion, our previous data have shown that $\mathrm{Bmx}$ is induced in keratinocytes and the Bmx-transgene specifically expressed in epidermal keratinocytes enhances wound reepithelialization (25).

The mechanisms by which Bmx mediates ischemia-induced arteriogenesis and angiogenesis are not clear. Mechanistically, ischemiainduced vascular remodeling occurring in both human patients and animal models includes the following distinct and function-dependent steps: hypoxia-induced activation of vascular endothelial cells; infiltration of inflammatory cells, particularly macrophages, which produce proinflammatory and proangiogenic factors; arterialization of preformed collaterals (arteriogenesis) in the upper limb; and capillary angiogenesis/vessel maturation in the lower limb. Defects in these steps could lead to failure of blood flow to tissue and recovery of functional limb salvage $(4,5)$. Our data suggest that $\mathrm{Bmx}$ contributes to multiple steps involved in ischemia-induced vascular remodeling. We have previously shown that $\mathrm{Bmx}$ associates with TNFR2 and VEGFR2 and mediates TNFR2- and VEGFR2-mediated EC migration and tube formation $(9,10)$. In this article, we showed that Bmx, TNFR2, and VEGFR2 were highly upregulated in ischemic hind limb and bone marrow. Most importantly, the expression and activation of TNFR2/VEGFR2 are Bmx dependent (i.e., are defective in Bmx-KO, whereas they are increased in Bmx-SK-Tg mice). These results suggest that $\mathrm{Bmx}$ is not only a downstream effector (activated by TNFR2 and VEGFR2), but also an upstream activator of TNFR2 and VEGFR2. This phenomenon has been reported for Bmx and VEGF signaling $(8,43)$. Consistent with our recent microarray analyses (25), we observed that $\mathrm{Bmx}$ is critical for gene expression of proinflammatory and proangiogenic molecules, likely via TNFR2and VEGFR2-dependent pathways. The exact mechanism by which Bmx expression/activation in ECs regulates gene expression of these molecules needs to be further investigated. Nevertheless, our study provides evidence supporting the hypothesis that Bmx-dependent TNFR2/VEGFR2 signaling is the principal mechanism by which $\mathrm{Bmx}$ mediates arteriogenesis/angiogenesis in vivo.

Our data clearly demonstrate that Bmx in bone marrow-derived cells plays a critical role in ischemia-induced vascular remodeling. This is supported by the results showing that bone marrow-derived cells from Bmx-KO and implanted into C57BL/ 6 mice impaired, whereas bone marrow-derived cells from Bmx-SK-Tg and implanted into C57BL/6 mice augmented, ischemia-induced blood flow recovery compared with those from WT mice. Bone marrow is the primary source of peripheral monocytes/macrophages and immune cells. Indeed, we observed that recruitment of macrophages $/ \mathrm{T}$ cells is significantly reduced in Bmx-KO mice but is dramatically augmented in Bmx-SK-Tg mice. Mobilization of EPCs from bone marrow to peripheral blood and injury sites has been proposed to account for the function of bone marrow cells (30-33). Whether or not $\mathrm{Bmx}$ regulates EPC mobilization in response to ischemia is under investigation. Interestingly, analyses of blood flow recovery in transplanted mice suggest that $\mathrm{Bmx}$ in bone marrow-derived cells is critical for the early phase of ischemia-induced tissue repair. Based on our analyses of inflammatory cells and gene expression profile, it is likely that Bmx/TNFR2/VEGFR2 signaling-dependent inflammatory/angiogenic responses mediate early effects of Bmx. Additionally, results of studies following BMT from WT to Bmx-KO mice suggest that $\mathrm{Bmx}$ in intrinsic vasculature contributes to the later phase of arteriogenesis/angiogenesis. Based on our in vitro findings $(9,10)$, it is conceivable that Bmx/TNFR2/VEGFR2dependent signaling in intrinsic ECs, by promoting migration, tube formation, and proliferation $(9,10)$, contributes to the later effects 
of $\mathrm{Bmx}$ in ischemic-induced arteriogenesis/angiogenesis in vivo. Given that Bmx is basally expressed in arterials (24), Bmx could also participate in the regulation of mechanical forces and shear stress, which are critical in shaping and/or remodeling collaterals or capillaries $(44,45)$. In support of this idea, our data indicate that Bmx deficiency reduces, whereas Bmx overexpression increases, postcontraction hyperemic responses, suggesting that $\mathrm{Bmx}$ regulates vasodilation and hymodynamics of arterial vessels.

Collectively, our data strongly support the role of Bmx in mediating ischemia-induced arteriogenesis and angiogenesis in vivo through multiple pathways by enhancing collateral growth, EPC mobilization, and angiogenesis, resulting in enhanced recovery of blood flow and vascular remodeling. Results of our study suggest that Bmx may be a novel target for the treatment of vascular diseases such as coronary artery and peripheral arterial diseases.

\section{Methods}

Generation of the Bmx-SK-Tg mice. The Tie-2 enhancer/promoter construct was from T.N. Sato (University of Texas Southwestern Medical Center at Dallas, Dallas, Texas, USA) (26). The human Bmx-SK cDNA with a Flag-tag sequence at the $5^{\prime}$ end was inserted into the NotI site between the Tie-2 promoter and enhancer. The plasmid was linearized with SalI digestion, and the transgenic mice were created by pronuclear injection of $\mathrm{C} 57 \mathrm{BL} / 6 \mathrm{~J} \times \mathrm{SJL} / \mathrm{J} \mathrm{F}_{2}$ embryos with the construct at Yale Transgenic Core. The founder transgenics were identified by PCR of tail DNA with a 5 ' primer of Flag and 3' primer of Bmx-SK.

Mouse hind limb ischemic model. All animal studies were approved by the Institutional Animal Care and Use Committee of Yale University. Eight- to 12-week-old male congenic $\left(\mathrm{F}_{10}\right) \mathrm{Bmx}-\mathrm{KO}, \mathrm{Bmx}-\mathrm{SK}-\mathrm{Tg}$ (bred in house at the Yale animal facility), or C57BL/6 mice (Jackson Laboratory) were used for all experiments. Mouse hind limb ischemia was induced as described previously (46). Briefly, following anesthesia $(79.5 \mathrm{mg} / \mathrm{kg}$ ketamine, $9.1 \mathrm{mg} / \mathrm{kg}$ xylazine), the left femoral artery was exposed under a dissection microscope. The proximal portion of the femoral artery and the distal portion of the saphenous artery were ligated. All branches between these 2 sites were ligated or cauterized, and arteriectomy was performed. Sham operations involved skin incision without femoral artery ligation.

Blood flow measurement and clinical score. Blood flow was measured by the Periflux System with Laser Doppler Perfusion module unit (LDPU; PERIMED). A deep measurement probe was placed directly on the gastrocnemius muscle to ensure a deep muscle flow measurement. Ischemic and nonischemic limb perfusion was measured before and directly after surgery and then 3 days, 2 weeks, and 4 weeks after surgery. The final blood flow values were expressed as the ratio of ischemic to nonischemic hind limb perfusion. To more precisely evaluate the mobility of mice after limb ischemia, we designed a scoring system: 0 , normal; 1 , pale foot (indicating reduced blood flow) or gait abnormalities; 2 , gangrenous tissue in less than half the foot without lower limb necrosis; 3 , gangrenous tissue in less than half the foot with lower limb necrosis; 4 , gangrenous tissue in more than half the foot; 5 , loss of half lower limb. Clinical outcome of all mice were observed and recorded at the same time points as blood flow measurement.

Postcontraction hyperemia before and after ischemia. After anesthesia, mice were placed on a heated pad. The adductor muscle group and gastrocnemius muscle were exposed by a midline incision of the limb. After baseline gastrocnemius blood flow was measured, adductor muscles were stimulated with 2 electrodes at $2 \mathrm{~Hz}, 5 \mathrm{~mA}$ using an electrostimulator for $2 \mathrm{~min}$ utes. Blood flow was taken and recorded by MacLab Chart software (ADInstruments) during stimulation and for 10 minutes after stimulation.

MICROFIL perfusion. Four weeks after femoral ligation, mice were anesthetized and perfused with $20 \mathrm{ml}$ of $37^{\circ} \mathrm{C}$ PBS plus $10 \mathrm{U} / \mathrm{ml}$ heparin at a flow rate of $10-15 \mathrm{ml} / \mathrm{min}$ through the left ventricle. After PBS, mice received $20 \mathrm{ml}$ of $4 \%$ paraformaldehyde and $15 \mathrm{ml}$ of MICROFIL (MV-112 [white]; Flow Tech Inc.). The MICROFIL polymerized overnight at $4{ }^{\circ} \mathrm{C}$, and the collagen gels and underlying abdominal musculature were harvested and clarified in graded glycerol solutions (40\%-100\% glycerol in water; glycerol increased by $20 \%$ at 24 -hour intervals). The clarified specimens were viewed on an SMZ1000 dissecting microscope (Nikon).

microCT analysis. Four weeks after femoral ligation, mice were anesthetized and perfused with $20 \mathrm{ml}$ of $37^{\circ} \mathrm{C}$ PBS plus $10 \mathrm{U} / \mathrm{ml}$ heparin at a flow rate of $10-15 \mathrm{ml} / \mathrm{min}$ through the left ventricle. To analyze the formation of new vasculature after onset of ischemia, we employed a silicone-based contrast formulation (MICROFIL; Flow Tech Inc.) to optimize visualization of the vascular structures in mice. All animals were imaged using the hybrid microSPECT/CT imaging system (X-SPECT; Gamma Medica-Ideas) implemented in the cone-beam geometry and equipped with a high-resolution GOS/CMOS detector achieving a resolution of less than $100 \mu \mathrm{m}$. The microCT detector was positioned at a distance of $64 \mathrm{~mm}$ from the center of rotation. High-resolution CT scans were performed using 512 projections, a $512 \times 512$ matrix, and $75 \mathrm{kV} / 250 \mu \mathrm{A}$ penetration energy. Scans with these parameters provide more than sufficient delineation of internal organs and the vasculature in proximal and distal hind limb. The contrast CT projection images were reconstructed using commercial software (COBRA; Exxim Computing Corp.) using a cone-beam optimized algorithm. The reconstructed images were visualized using $3 \mathrm{D}$ rendering with Amira software (Indeed - Visual Concepts $\mathrm{GmbH}$ ). For qualitative analysis of vasculature, the bone was manually wiped, and vasculature volume was quantified by image analyses using NIH Image J (http://rsb.info.nih.gov/ij/).

Histology and immunohistochemistry. Mice were sacrificed at 4 weeks after surgery, and muscles of the lower limbs were harvested, methanol fixed, and paraffin embedded. Tissue sections ( $5 \mu \mathrm{m}$ thick) were stained using anti-Bmx antibody (C17; Santa Cruz Biotechnology Inc.), anti-PECAM-1 antibody (BD Biosciences - Pharmingen) and anti- $\alpha$-SMA antibody (Dako). Bound primary antibodies were detected using avidin-biotinperoxidase (NovaRED Peroxidase Substrate Kit; Vector Laboratories). Pictures from 4 random areas of each section and 5 sections per mice were taken using a Kodak digital camera mounted on a light microscope ( $\times 40$ objective). Capillary density and $\alpha$-SMA positivity were quantified by measuring the percentage of PECAM-positive area or $\alpha$-SMA-positive area (in same area of adjacent sections) out of the total area using MATLAB software (The MathWorks).

Gene expression in ischemic muscle. Total RNA of lower limb muscles was isolated by using phenol/chloroform and isolated using RNeasy kit with DNase I digestion (QIAGEN). Reverse transcription was done using a standard procedure (SuperScript First-Strand Synthesis System; Invitrogen) using $1 \mu \mathrm{g}$ total RNA. qRT-PCR was performed by using iQ SYBR Green Supermix on iCycler Real-Time Detection System (Bio-Rad). Specific primers for Ang II, Tie-2, PlGF, VEGFR2 (Flk-1), TNF, TNFR1, TNFR2, VCAM-1, TRAF2, and PDGFR- $\beta$ were used. 18 S ribosomal RNA was used as an internal control. The relative amount of mRNA in lower limb muscles was quantified. Phospho- and total Bmx, phospho- and total VEGFR2, and TNFR2 proteins were determined by Western blotting as described previously (9). Anti-phospho-Bmx antibody (pY40) was from Cell Signaling Technology.

$B M T$. All animal studies were approved by the institutional animal care and use committees of Helsinki University. BMT was performed in lethally irradiated mice (irradiated twice at $5.5 \mathrm{~Gy}$ ) within 3 hours) using cells derived from donor mice, and bone marrow cells from WT, Bmx-KO, or Bmx-SK-Tg mice were harvested by flushing femur. Red cells were removed by lysis using ammonium chloride and subsequent washing with PBS. Cells were counted, and $1 \times 10^{5}$ cells were injected into the tail vein of the recipient. 
Successful BMT was controlled by FACS analysis of peripheral blood or by genotyping of Bmx gene 6 weeks after BMT. The mice were then subjected to femoral artery ligation as described above. Complete blood tests were performed by Antech Diagnostics.

Statistics. All data are expressed as mean \pm SEM. Statistical differences were measured by the nonparametric Mann-Whitney $U$ test. A value of $P<0.05$ was considered statistically significant.

\section{Acknowledgments}

This work was supported in part by an Established Investigator Award from the American Heart Association and NIH grant R01HL65978-5 to W. Min and grants from the "973" National Basic Key Research and Development Program and the National
Natural Science Foundation of China to Y. He. We thank Wawrzyniec L. Dobrucki for assistance on the microCT analysis.

Received for publication February 6, 2006, and accepted in revised form June 27, 2006.

Address correspondence to: Wang Min, Interdepartmental Program in Vascular Biology and Transplantation, Department of Pathology, Yale University School of Medicine, BCMM 454, 295 Congress Avenue, New Haven, Connecticut 06510, USA. Phone: (203) 785-6047; Fax: (203) 737-2293; E-mail:wang.min@yale.edu.

Yun He and Yan Luo contributed equally to this work.
1. Baumgartner, I., Schainfeld, R., and Graziani, L. 2005. Management of peripheral vascular disease. Annu. Rev. Med. 56:249-272.

2. Mohler, E.R., 3rd. 2004. The effect of risk factor changes on peripheral arterial disease and cardiovascular risk. Curr. Drug Targets Cardiovasc. Haematol. Disord. 4:259-263.

3. Brevetti, G., et al. 2003. Endothelial dysfunction in peripheral arterial disease is related to increase in plasma markers of inflammation and severity of peripheral circulatory impairment but not to classic risk factors and atherosclerotic burden. J. Vasc. Surg. 38:374-379.

4. Heil, M., and Schaper, W. 2004. Influence of mechanical, cellular, and molecular factors on collateral artery growth (arteriogenesis). Circ. Res. 95:449-458.

5. Heil, M., and Schaper, W. 2005. Cellular mechanisms of arteriogenesis. EXS. 94:181-191.

6. Tamagnone, L., et al. 1994. BMX, a novel nonreceptor tyrosine kinase gene of the BTK/ITK/TEC/TXK family located in chromosome Xp22.2. Oncogene. 9:3683-3688.

7. Qiu, Y., Robinson, D., Pretlow, T.G., and Kung, H.J. 1998. Etk/Bmx, a tyrosine kinase with a pleckstrinhomology domain, is an effector of phosphatidylinositol 3'-kinase and is involved in interleukin 6-induced neuroendocrine differentiation of prostate cancer cells. Proc. Natl. Acad. Sci. U. S. A. 95:3644-3649.

8. Chau, C.H., et al. 2002. Coordinated Etk/Bmx activation and VEGF upregulation to promote cell survival and proliferation. Oncogene. 21:8817-8829.

9. Zhang, R., et al. 2003. Etk/Bmx transactivates vascular endothelial growth factor 2 and recruits phosphatidylinositol 3-kinase to mediate the tumor necrosis factor-induced angiogenic pathway. J. Biol. Chem. 278:51267-51276.

10. Pan, S., et al. 2002. Etk/Bmx as a tumor necrosis factor receptor type 2 -specific kinase: role in endothelial cell migration and angiogenesis. Mol. Cell. Biol. 22:7512-7523.

11. Tsai, Y.T., et al. 2000. Etk, a Btk family tyrosine kinase, mediates cellular transformation by linking Src to STAT3 activation. Mol. Cell. Biol. 20:2043-2054.

12. Mao, J., et al. 1998. Tec/Bmx non-receptor tyrosine kinases are involved in regulation of Rho and serum response factor by Galpha12/13. EMBO J. 17:5638-5646.

13. Chen, R., et al. 2001. Regulation of the PH-domaincontaining tyrosine kinase Etk by focal adhesion kinase through the FERM domain. Nat. Cell Biol. 3:439-444.

14. Qiu, Y., and Kung, H.J. 2000. Signaling network of the Btk family kinases. Oncogene. 19:5651-5661.

15. Kim, O., Yang, J., and Qiu, Y. 2002. Selective activation of small GTPase RhoA by tyrosine kinase Etk through its pleckstrin homology domain. J. Biol. Chem. 277:30066-30071.
16. Bagheri-Yarmand, R., et al. 2001. Etk/Bmx tyrosine kinase activates Pak1 and regulates tumorigenicity of breast cancer cells. J. Biol. Chem. 276:29403-29409.

17. Kiosses, W.B., et al. 2002. A dominant-negative p65 PAK peptide inhibits angiogenesis. Circ. Res. 90:697-702.

18. Qi,J.H., and Claesson-Welsh, L. 2001. VEGF-induced activation of phosphoinositol 3-kinase is dependent on focal adhesion kinase. Exp. Cell Res. 263:173-182.

19. Gille, H., et al. 2000. A repressor sequence in the juxtamembrane domain of Flt-1 (VEGFR-1) constitutively inhibits vascular endothelial growth factor-dependent phosphatidylinositol 3'-kinase activation and endothelial cell migration. EMBOJ. 19:4064-4073.

20. Dimmeler, S., Dernbach, E., and Zeiher, A.M. 2000 Phosphorylation of the endothelial nitric oxide synthase at Ser-1177 is required for VEGF-induced endothelial cell migration. FEBS Lett. 477:258-262.

21. Kureishi, Y., et al. 2000. The HMG-CoA reductase inhibitor simvastatin activates the protein kinase Akt and promotes angiogenesis in normocholesterolemic animals. Nat. Med. 6:1004-1010.

22. Ackah, E., et al. 2005. Akt1/protein kinase B $\alpha$ is critical for ischemic and VEGF-mediated angiogenesis. J. Clin. Invest. 115:2119-2127. doi:10.1172/JCI24726.

23. Matsumoto, T., and Claesson-Welsh, L. 2001. VEGF receptor signal transduction. Sci. STKE. 112:RE21.

24. Rajantie, I., et al. 2001. Bmx tyrosine kinase has a redundant function downstream of angiopoietin and vascular endothelial growth factor receptors in arterial endothelium. Mol. Cell. Biol. 21:4647-4655.

25. Paavonen, K., et al. 2004. Bmx tyrosine kinase transgene induces skin hyperplasia, inflammatory angiogenesis, and accelerated wound healing. $\mathrm{Mol}$. Biol. Cell. 15:4226-4233.

26. Schlaeger, T.M., et al. 1997. Uniform vascularendothelial-cell-specific gene expression in both embryonic and adult transgenic mice. Proc. Natl. Acad. Sci. U. S. A. 94:3058-3063.

27. Bauer, P.M., et al. 2005. Endothelial-specific expression of caveolin-1 impairs microvascular permeability and angiogenesis. Proc. Natl. Acad. Sci. U. S. A. 102:204-209.

28. Yu, J., et al. 2005. Endothelial nitric oxide synthase is critical for ischemic remodeling, mural cell recruitment, and blood flow reserve. Proc. Natl. Acad. Sci. U. S. A. 102:10999-11004.

29. Arras, M., et al. 1998. Monocyte activation in angiogenesis and collateral growth in the rabbit hindlimb. J. Clin. Invest. 101:40-50.

30. Takahashi, T., et al. 1999. Ischemia- and cytokineinduced mobilization of bone marrow-derived endothelial progenitor cells for neovascularization. Nat. Med. 5:434-438.

31. Asahara, T., et al. 1999. VEGF contributes to postnatal neovascularization by mobilizing bone mar- row-derived endothelial progenitor cells. EMBO J. 18:3964-3972.

32. Aicher, A., et al. 2003. Essential role of endothelial nitric oxide synthase for mobilization of stem and progenitor cells. Nat. Med. 9:1370-1376.

33. Hristov, M., Erl, W., and Weber, P.C. 2003. Endothelial progenitor cells: mobilization, differentiation, and homing. Arterioscler. Thromb. Vasc. Biol. 23:1185-1189.

34. Carmeliet, P., et al. 2001. Synergism between vascular endothelial growth factor and placental growth factor contributes to angiogenesis and plasma extravasation in pathological conditions. Nat. Med. 7:575-583.

35. Silvestre, J.S., et al. 2000. Antiangiogenic effect of interleukin-10 in ischemia-induced angiogenesis in mice hindlimb. Circ. Res. 87:448-452.

36. Sasaki, K., et al. 2002. Evidence for the importance of angiotensin II type 1 receptor in ischemiainduced angiogenesis. J. Clin. Invest. 109:603-611. doi:10.1172/JCI200213055.

37. Johnson, C., Sung, H.J., Lessner, S.M., Fini, M.E., and Galis, Z.S. 2004. Matrix metalloproteinase-9 is required for adequate angiogenic revascularization of ischemic tissues: potential role in capillary branching. Circ. Res. 94:262-268.

38. Shibata, R., et al. 2004. Adiponectin stimulates angiogenesis in response to tissue ischemia through stimulation of amp-activated protein kinase signaling. J. Biol. Chem. 279:28670-28674.

39. Sonveaux, P., et al. 2004. Caveolin-1 expression is critical for vascular endothelial growth factor-induced ischemic hindlimb collateralization and nitric oxidemediated angiogenesis. Circ. Res. 95:154-161.

40. Heil, M., et al. 2004. Collateral artery growth (arteriogenesis) after experimental arterial occlusion is impaired in mice lacking CC-chemokine receptor-2. Circ. Res. 94:671-677.

41. Tang, G., Charo, D.N., Wang, R., Charo, I.F., and Messina, L. 2004. CCR2-/- knockout mice revascularize normally in response to severe hindlimb ischemia. J. Vasc. Surg. 40:786-795.

42. Murohara, T., et al. 1998. Nitric oxide synthase modulates angiogenesis in response to tissue ischemia. J. Clin. Invest. 101:2567-2578.

43. Chau, C.H., et al. 2005. Etk/Bmx mediates expression of stress-induced adaptive genes VEGF, PAI-1, and iNOS via multiple signaling cascades in different cell systems. Am. J. Physiol. Cell Physiol. 289:C444-C454.

44. Hudlicka, O. 1991. What makes blood vessels grow? J. Physiol. 444:1-24.

45. Le Noble, F., et al. 2004. Flow regulates arterialvenous differentiation in the chick embryo yolk sac. Development. 131:361-375.

46. Couffinhal, T., et al. 1998. Mouse model of angiogenesis. Am. J. Pathol. 152:1667-1679. 